\title{
Ternary coherent phase diagram on the FCC lattice
}

\author{
P. Cénédèse $\left({ }^{1}\right)$, Y. Calvayrac $\left({ }^{1}\right)$ and A. Marty $\left({ }^{2}\right)$ \\ (1) CECM/CNRS, 15 rue G. Urbain, 94407 Vitry-sur-Seine, France \\ ( ${ }^{2}$ ) CENG/DRFMC/SPMM/MP, 85X, 38041 Grenoble Cedex, France \\ (Received 28 January 1994, recerved in final form 25 March 1994, accepted 30 March 1994)
}

\begin{abstract}
In this paper, we describe a general and original method to study the ground state problem, in multicomponent systems. The method is illustrated by considering the nearest neighbour Ising model in ternary FCC lattices, and leads to the occurrence as a ground state of a new phase called $L^{\prime}$. The ternary coherent phase diagram is then calculated within the CVM tetrahedron approximation, showing that the $\mathrm{L}^{\prime}$ phase displays a stability domain as large as those of the $\mathrm{L} 1_{0}$ and $\mathrm{Ll}_{2}$ domains. At low but non-zero temperature, a * quaternary ground state » phase, called L", stabilized by entropy effects, appears in the phase diagram.
\end{abstract}

\section{Introduction.}

Alloy design deals generally with multicomponent systems, while a great amount of experimental and theoretical studies are available mostly for binary mixtures. This may come from the fact that the work involved in $m$-component systems exponentially grows with $m$, and ternary alloys might be considered as the first step to be made in that field.

Prediction of phase diagram patterns can be achieved by either using (semı) empirical methods or using first principles calculations.

In the first scheme, empiric models with a few parameters are used. The model parameters are then fitted from the avallable experimental information or data bank. The main advantage of this scheme is to digitalize the phase diagram information, notably in multi-component systems where, owing to the lack of graphical mapping, we are somewhat blind. These methods are also helpful in predictıng phase diagram topology from a minimal set of experıments. These methods have some drawbacks too, as they cannot predict the occurrence of new phases, and the transferability of information from problem to problem might be a severe limitation.

The other scheme would follow first principle calculations lines, combining ab intto band structure computations together with improved statistical models such as the Monte Carlo method or the Cluster Variation Method [1]. Recent works in this promising field have been done in ternary alloys [2]. The main problem raised by this theoretical approach of phase alloy design, deals with the functional form of the interaction potentials which can be concentration 
dependent effective pair wise interactions [3] (which can be coherently « measured " from diffuse scattering experiments [4]) or can include many body interactions [5]. Here also recent progress has been achieved [6].

It is one of the two goals of the present paper to show that, independently of electronic structure calculations, the CVM may give insight into the topology of ternary phase diagram. A first attempt to classify stabilities of the ternary phases was proposed by Meijering [7]. Using the regular solution model, Meijermn [7] classified ternary solid solutions (ABC) into eight categories. Meijering [7] divided the regular ternary solid solutions into four classes following the sign of the interactions $J_{\mathrm{AB}}, J_{\mathrm{AC}}, J_{\mathrm{BC}}$, splitting each class into two subsets according to the sign of the parameter $J_{\mathrm{AC}}-J_{\mathrm{BC}}$. The problem of miscibility gap and spinodal surface was revisited by Kikuchi et al. [8], and Kikuchi [9] considered the case of the Au-Ag$\mathrm{Zn}$ system, but to our knowledge the case where the three binary pair interactions correspond to ordering was not considered in detall.

The second goal of this paper is to describe a new algorithm to solve the ground state analysis problem or $0 \mathrm{~K}$ phase diagram.

\section{Ground states analysis.}

2.1 A GENERAL METHOD. - As suggested in the introduction, the CVM approximation is especially well designed to describe first order transitıons in alloys, where the configurational entropy is the leading factor. The precise design of a phase diagram is essentially constrained by the given intermetallic compounds considered in the computations. The knowledge of the plausible ordered phases occurring at $0 \mathrm{~K}$, known as the ground state analysis problem (GSA) is therefore of a major interest. Furthermore, the knowledge of the stable phases at $0 \mathrm{~K}$ gives the clue to the prediction of the phase diagram topology at higher temperatures $T$ in the chemical potentral $\mu$ space.

In addition to these general considerations we would like to mention that in its own the GSA gives invaluable information to scale first principle calculations. Indeed, for a given parent lattice and for a given functional form of the potentials between atoms, the GSA gives bounds on the magnitude of the interactions stabilizing a given intermetallic.

Some theoretical methods have been devised to solve the GSA problem, which have mostly been applied in the field of binary mixtures. These methods belong to three main techniques, which we do not review in detall.

The first method is due to Kanamorı [10], and Kanamorı and Kaburag1 [11]. It consısts in definıng geometrical inequalities by using spin operators from the consideration of cluster configuration probabilities. The method is strongly dependent on the functional form of the internal energy.

The second way developed by Allen and Cahn [12], and Cahn and Kikuchi [13], may handle multiplet interactions in addition to pair interactions. The method uses a redundant cluster probability basis for fixed alloy compositions, but in its primitıve description it is restrıcted to simplex clusters only.

The third method, due to Finel [14], uses a global description of the solutions of the GSA in the context of the vertices of the convex polyhedron $\mathfrak{T}_{\alpha}$ of a cluster $\alpha$. The convex hull of the projection of these vertices in the subspace of the correlation functions associated with nonzero energies is then computed and its dual polyhedron $\tilde{\mathfrak{S}}_{\alpha}$ in the subspace of the energy parameters is obtained.

Even if all these briefly discussed methods can be extended to the context of ternary alloys, we would like to devote the end of this section to the description of a different method which is entirely based on our ability to solve homogeneous linear system of inequalitıes, referred to hereafter as the problem I. 
The difficulty raised by considering $m$ components rather than binary alloys is wholly hidden in the dimension of the various matrices we will consider, but without any other special character. As we will see, the ground state analysis problem is fully and simply handled by applying three times consecutively the mathematically clean algorithm of Motzkin and Burger [15] for solving problem I. Let us remind the algorithm :

Let $A$ be a rectangular matrix belonging to $\mathbb{R}^{m} \times \mathbb{R}^{n}$ where $m>n$, also considered as an assembly of $m$ row vectors $\mathbf{a}_{1}, \mathbf{a}_{2} ; \ldots ; \mathbf{a}_{m}$ of dimension $\mathbb{R}^{n}$. Problem $\mathrm{I}$ consists in determining all the solution vectors $\mathbf{X}$ of $\mathbb{R}^{n}$ such that :

$$
A \mathbf{X} \geqslant 0 \text {. }
$$

Any solution $\mathbf{X}$ can be written as a positive linear combination of $n_{\mathrm{A}}$ cone basis vectors $\mathbf{u}$ of $\mathbb{R}^{n}$, that can be found by using the algorithm of Motzkin and Burger. We have :

$$
\begin{aligned}
& \mathbf{X}=\sum_{,=1}^{\prime=n_{\mathrm{A}}} \lambda_{1} \mathbf{u}_{1} \\
& \forall i \quad \lambda_{1} \geqslant 0 \\
& \forall i \quad A \mathbf{u}_{i} \geqslant \mathbf{0} .
\end{aligned}
$$

In problem $1, A$ is a full rank matrix of order $n$, so we safely assume that its first $n$ row vectors $\mathbf{a}_{1}$ to $\mathbf{a}_{n}$ are linearly independent (we can always pre-multiply the matrix $A$ by $a$ $\mathbb{R}^{m} \times \mathbb{R}^{m}$ permutation matrix). Starting from the square matrix $\tilde{A}$ of order $n$ associated with these vectors, we define exactly $n$ cone vectors $\left(\mathbf{u}_{1}: \mathbf{u}_{2}, \ldots, \mathbf{u}_{n}\right)_{0}$ as the column vectors associated with the matrix $\tilde{A}^{-1}$ (or $\tilde{A}^{-1} D$ where $D$ is a positive definite matrix of order $n$ ).

From the set of cone vectors $\{\mathbf{u}\}_{0}$, we form a new set $\{\mathbf{u}\}_{1}$ taking the next inequality into account. This new set of basis vectors includes those vectors of $\{\mathbf{u}\}_{0}$ that satisfy the inequality $\mathbf{a}_{n+1} \cdot\left(\mathbf{u}_{1}\right)_{0} \geqslant 0$, and some new vectors defined from any couple of vectors $\left(\mathbf{u}_{1}\right)_{0}$ and $\left(\mathbf{u}_{j}\right)_{0}$ that exactly annihilate simultaneously $(n-2)$ previous inequalities and such that :

$$
\mathbf{u}=\left|\mathbf{a}_{n+1} \quad\left(\mathbf{u}_{j}\right)_{0}\right|\left(\mathbf{u}_{l}\right)_{0}+\left|\mathbf{a}_{n+1} \cdot\left(\mathbf{u}_{2}\right)_{0}\right|\left(\mathbf{u}_{j}\right)_{0}\left(\mathbf{a}_{n+1} \quad\left(\mathbf{u}_{\imath}\right)_{0}\right) \times\left(\mathbf{a}_{n+1} \quad\left(\mathbf{u}_{j}\right)_{0}\right)<0 .
$$

Starting from this new set of cone vectors, the scheme is repeated untll all the non-redundant inequalities have been considered.

We now demonstrate how problem I naturally happens in the GSA problem, and in the following subsection we shall illustrate the method in the case of the nearest neighbour FCC ternary Ising model, postponing the study of more complicated cases to a later publication.

At zero temperature the minimization problem of $\mathcal{F}$ reduces to the minimization of the internal energy $u$, becoming a linear programming problem which is expected to be easier to solve. In the Ising model, the internal energy written :

$$
U=\sum_{i}^{\prime} \sum_{i j} V_{i j}(r) P_{i j}(r)+\sum_{1} \mu, C_{l}
$$

Where $r$ indexes the neighboring shells and runs up to $r_{\max }$ defined as the (short) range of the pair interactions.

The indices $i, j$ label the atomic species and run from 1 to $m$, where $m$ is the number of components of the alloy.

Two atoms $l$ and $j$ which are, -th neighbours interact through an energy parameter $V_{i j}(r)$ with the probability $P_{i j}(r)$. 
The concentration of atom $i$ in the solution 1 driven by the chemical potential $\mu_{1}$. As the sum of the concentrations equals 1 , only $m-1$ chemical potentials are independent, thus without loss of generality we set:

$$
\sum_{i=1}^{m} \mu_{i}=0 .
$$

The linear expression (1), well suited to the context of pair interactions, is a special feature of the general linear form :

$$
U=\sum_{h} J_{h} X_{h}=\mathbf{J} \cdot \mathbf{X}
$$

In expression (2), $k$ runs on the $n+1$ components of the vector $\mathbf{X}$, known as the correlation functions set. With each component $X_{k}$ of $\mathbf{X}$, we associate a conjugated variable $J_{h}$ of $\mathbf{J}$, that is energy parameter. For convenience the components of $\mathbf{X}$ are sorted according to a sequence of increasing sized clusters. As a rule, the zero component of $\mathbf{X}$ is the empty correlation function associated with the normalization constraint on probabilities, the $(m-1)$ next components of $\mathbf{X}(\operatorname{resp} \mathbf{J})$ refer to the $(m-1)$ point correlation functions (resp. chemical potentials), the following $\frac{m(m-1)}{2}$ components are the pair correlation functions (resp. effective pair energies). The remaining components are multıplets and simılar energies are defined. The components of $\mathbf{X}$ are independent variables and form a basis to describe the state of order of a basic cluster $\alpha$ (or any linear combination of basic clusters), whose size and shape encompass all the non-zero interactions.

In the disordered phase let $A_{\alpha}$ be the matrix linking the $n_{\alpha}$ dimensional vector $\mathbf{P}_{u}$, (the components of which label the various ways of decorating the cluster $\alpha$ ), to the correlation function vector $\mathbf{X}$. We have thus :

$$
\begin{gathered}
\mathbf{P}_{\alpha}=A_{\alpha} \mathbf{X} \\
\mathbf{P}_{\alpha} \in \mathbb{R}^{n_{\alpha}} ; \quad \mathbf{X} \in \mathbb{R}^{n+1} \\
A_{\alpha} \in \mathbb{R}^{n_{\alpha}} \times \mathbb{R}^{n+1}
\end{gathered}
$$

The ground states have to be determined among the configurations of the cluster $\alpha$ which minımızes the internal energy (2) submitted to the constraints :

$$
\mathbf{P}_{\alpha}=A_{\alpha} \mathbf{X} \geqslant \mathbf{0}
$$

The extremum of the energy $U$ falls on the vertices of the linear system of inequalities (3), which have the standard form of problem $I$, thus we have :

$$
\begin{gathered}
\mathrm{X}=\sum_{t=1}^{\prime=n_{\mathrm{c}}} \lambda_{l} \boldsymbol{v}_{1} \\
\forall v_{1}: A_{\alpha} \boldsymbol{v}_{1} \geqslant 0 \\
\lambda_{1} \geqslant 0 .
\end{gathered}
$$

In our formalism the constraints have defined cone vectors in $\mathbb{R}^{n+1}$ while they define convex polyhedra $\mathfrak{T}_{\alpha}$ in method 3. The link between the two methods is obvious as we define directions in $\mathbb{R}^{n}$, thus we may safely normalize the always strictly positive zeroth component of each cone vector to unity. 
Up to now, the two ways are equivalent, except for the method followed to find the vertices, where we have handled the inequalities straightforwardly and in the simplest possible way. The main difference arises in the following steps of the analysis.

The general statement of the GSA problem is to find the conditions holding on the set of the pair interactions $\mathbf{J}$ which in some domain of concentrations, stabilize a given phase associated with one of the $n_{\mathrm{c}}$ vectors $\boldsymbol{v}$.

Starting from equation (2), we associate an energy vector $\mathbf{U}_{\left(v_{1}\right)}$ with each cone vector. This vector of dimension $n_{u}$ is defined as the projection of the cone vector $v_{l}$ onto the subspace of the non-zero energy parameters. For example in the case of pair wise interactions, $n_{u}=(m-1)+r_{\max } \frac{m(m-1)}{2}$ and the components of $\mathbf{U}_{\left(v_{1}\right)}$ are the $m-1$ components of the cone vector $v_{1}$ corresponding to the point correlation functions while $r_{\max } \frac{m(m-1)}{2}$ comes from the pair correlation function components of $v_{l}$. The corresponding configuration of the cluster $\alpha$ would be stabilized if its energy were lower than the energy of all other competing configurations. Collecting under a matrix form the energy differences between a given phase labelled $i_{0}$ with all the others, we have :

$$
\begin{gathered}
\forall j \neq i_{0} \quad\left(\mathbf{U}_{\left(\boldsymbol{v}_{j}\right)}-\mathbf{U}_{\left(\nu_{r_{0}}\right)}\right) \cdot \mathbf{J} \geqslant 0 \\
\sum_{h=1}^{h=n_{u}}\left(\nu_{j}^{k}-\nu_{i_{0}}^{h}\right) J_{h} \geqslant 0 \\
B_{i_{0}} \mathbf{J} \geqslant \mathbf{0}
\end{gathered}
$$

where $B_{i_{0}}$ is a $\mathbb{R}^{n_{r}-1} \times \mathbb{R}^{n_{u}}$ matrix.

The statement involved in the system of inequalities (5), is once again a standard problem $I$. Let $\kappa$ denote the $n_{u}$ dimensional cone vectors of (5). At this stage the ground state analysis can be considered as almost ended. The final step we now consider is essentially a matter of data representation.

If we adopt a vector picture of the GSA, it is sufficient to give the components of the $n_{\kappa}$ vectors $\kappa$ together with the meaning of each component (point, pair, triangle, etc...). The interested reader would have then to check that, for the physical problem considered, the associated energy parameters can be written :

$$
\begin{aligned}
& \mathbf{J}=\sum_{i=1}^{i=n_{\kappa}} \lambda_{1} \boldsymbol{\kappa}_{i} \\
& \forall i \quad \lambda_{i} \geqslant 0 .
\end{aligned}
$$

The first $(m-1)$ components of $\boldsymbol{\kappa}$, are associated with the point correlation functions and are irrelevant in the problem. Indeed, we are allowed to choose the composition of the alloy, which amounts to scanning the chemical potential space, and as a matter of fact the $(m-1)$ first constraints of $(6)$ are necessarily satisfied by the $(m-1)$ first components of $\mathbf{J}$. It is customary to present the results of GSA problem as a set of inequalities holding between the interactions, and to map them onto two-dimensional plots. The first task can be easily performed by applying the formalısm once again. By removing the first $(m-1)$ components of $\mathrm{J}$, equation (6) is written once more under the matrix form :

$$
\tilde{\mathbf{J}}=C \boldsymbol{\lambda}
$$


where $\tilde{\mathbf{J}}$ is the projection of the vector $\mathbf{J}$ in the subspace of non-zero parr, triangle, etc..., interactions of dimensionality $\tilde{n}_{u}=n_{u}-(m-1)$. $C$ is the matrix formed from the components of the vectors $\boldsymbol{\kappa}$ in this subspace, thus $C \in \mathbb{R}^{\tilde{n}_{u}} \times \mathbb{R}^{n_{\star}}$ and $\boldsymbol{\lambda}$ is a semi-positive definite vector in $\mathbb{R}^{n_{n}}$.

The solution of the linear system (Eq. (7)) is possible if, and only if, the following condition 1s obeyed :

$$
\forall \mathbf{y} \in \mathbb{R}^{\tilde{n}_{u}} \quad \text { such that } \quad \mathbf{y} C \geqslant \mathbf{0} \Rightarrow \mathbf{y} \tilde{\mathbf{J}} \geqslant \mathbf{0} .
$$

The above existence condition (8) for the positive solution of (7) is due to Farkas and Minkovski [16]. We now note that (8) is simılar to the system of inequalities we have already solved, provided that we transpose both matrix and vector. In this way we end up with new cone vectors that are just the usual inequalities holding between the interactions.

At this stage, the formalism is general and many-body interactions included in the maximum cluster have been taken into account. In practice the method is very easy to work out and very efficient as it is sufficient to consider three successive applications of the standard MotzkinBurger [15] algorithm for solving homogeneous linear systems of inequalities.

The GSA problem is now completely solved. However, for the GSA to be complete, there remains (as in all other methods) to make a one-to-one correspondence between the ground state cone vectors and lattice structures.

2.2 Ternary FCC GRound STATEs. - We now illustrate the method in the case of the nearest neighbour Ising model in ternary FCC solutions $\left({ }^{1}\right)$. As in related studies of binary alloys, in order to handle the geometrical lattice frustration we choose the regular tetrahedron to be the cluster $\alpha$. This cluster is a simplex and the matrix $A$ linking the tetrahedron probabilities $\mathbf{P}_{\alpha}$ of the disordered phase to the correlation functions $\mathbf{X}$ is a square matrix of order 15 . The cone vectors $\boldsymbol{v}$ correspond to the column vectors of the inverse of the matrix $A$, and to each vector $\boldsymbol{v}$ is associated a given FCC ordered phase. In this model, which is the simplest for orderıng problems, we deal with three pair orderıng energies:

$$
\begin{aligned}
& J^{\mathrm{AB}}=\frac{V^{\mathrm{AA}}+V^{\mathrm{BB}}-2 V^{\mathrm{AB}}}{4} \\
& J^{\mathrm{AC}}=\frac{V^{\mathrm{AA}}+V^{\mathrm{CC}}-2 V^{\mathrm{AC}}}{4} \\
& J^{\mathrm{BC}}=\frac{V^{\mathrm{BB}}+V^{\mathrm{CC}}-2 V^{\mathrm{BC}}}{4}
\end{aligned}
$$

Projectung this set of equations into the 3-dimensional space of pair energies, all the $B$ matrices we can form are full rank matrices of dimension 14 by 3 , the cone vectors of which are solutions of the GSA.

As long as the entropy is not involved, the scaling of the pair energies by a common positive factor does not affect the state of order. The stability ranges of each phase in the space $\mathbf{J}=\left(J^{\mathrm{AB}}, J^{\mathrm{AC}}, J^{\mathrm{BC}}\right)$ are cone vectors meetıng at the origin. Thus only two parameters are independent allowing then to map the results onto a surface. This surface could be a sphere, but we favor the representation of the direction of $\mathbf{J}$ on two planes which preserve the ternary

(1) The method can be applied to the case of next nearest neighbour interactions. However we should be aware that the work grows exponentially because the number of cone vectors becomes extremely large and it is unlikely to map each candidate ground state in a one to one correspondence with an FCC structure. 
symmetry. These planes correspond to the cut of the Euclidian 3-dimensional space perpendicular to the main diagonal defined by : $J^{\mathrm{AB}}+J^{\mathrm{AC}}+J^{\mathrm{BC}}= \pm 1$

The plane $J^{\mathrm{AB}}+J^{\mathrm{AC}}+J^{\mathrm{BC}}=-1$ does not present any interest for our purpose since it deals only with the binary ordered phases and clustering.

More interesting is the upper plane $J^{\mathrm{AB}}+J^{\mathrm{AC}}+J^{\mathrm{BC}}=1$, in which appear three new phases which are ground states in ternary but not in binary alloys. These $A_{2} B C$ type phases are superstructures with three different sublattıces that are built by tilıng the FCC lattice with only one kind of tetrahedra (see Tab. I). Such a structure is a superstructure of both the $\mathrm{Ll}_{2}$ and $\mathrm{L} 1_{0}$ structures, and $1 \mathrm{~s}$ degenerated with all the structures differing through conservatives $\frac{\langle 110\rangle}{2}$ APB. Following Finel and Ducastelle [17], we will call this structure $L^{\prime}$. In figure 1 , we have mapped the conditions of stability of one of these ground states in a system of barycentric coordinates with respect to the three points $\mathbf{J}=(1,0,0),(0,1,0)$, $(0,0,1)$.

Table I. - The different structures occuring $m$ the phase diagram of the nearest neighbour Ising model of the ternary FCC lattice.

\begin{tabular}{|c|c|c|}
\hline $\begin{array}{c}\text { Tetrahedron } \\
\text { state }\end{array}$ & $\begin{array}{c}\text { Structure } \\
\text { Space group } \\
\text { Sublattice } \\
\text { equivalence } \\
\text { Ground state }\end{array}$ \\
\hline Cubic cell \\
Fm3m \\
I=II III = IV \\
A (pure)
\end{tabular}




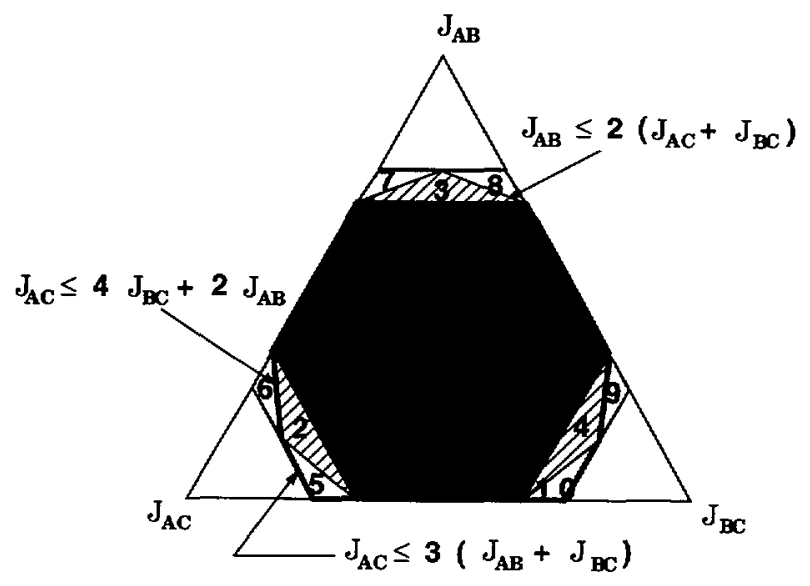

Fig. 1. - Isometric representation of the stability conditions of the $L^{\prime}$ phases. The three vertices of the triangle correspond to $\mathbf{J}=(1,0,0), \mathbf{J}=(0,10)$ and $\mathbf{J}=(0,0,1)$. The $\mathrm{A}_{2} \mathrm{BC}$ phase is found to be stable inside the domains labelled $12,4,5$ and $10 .\left(A_{2} C\right.$ corresponds to $13,4,8$ and 9 and $\mathrm{ABC}_{2}$ to $1,2,3,6$ and 7.)

The $\mathrm{A}_{2} \mathrm{BC}$ phase is only stable within a polygon inside the triangle of positıve pair energies, which means that the three pair energies have to favor ordering. Moreover, these energies should be close to each other: they must obey the following system :

$$
\begin{gathered}
J^{\mathrm{AB}} \leqslant 3 J^{\mathrm{AC}}+3 J^{\mathrm{BC}} ; \quad J^{\mathrm{AC}} \leqslant 3 J^{\mathrm{AB}}+3 J^{\mathrm{BC}} \\
J^{\mathrm{AB}} \leqslant 4 J^{\mathrm{AC}}+2 J^{\mathrm{BC}} ; \quad J^{\mathrm{AC}} \leqslant 4 J^{\mathrm{AB}}+2 J^{\mathrm{BC}} \\
J^{\mathrm{BC}} \leqslant 2 J^{\mathrm{AB}}+2 J^{\mathrm{AC}}
\end{gathered}
$$

By exchanging the species A, B and C, the conditions of stability of the two other phases may be obtained.

However, the domain of stability is large enough for the possible existence of such ordered phases in real ternary alloys. This seems to be the case for the ternary alloy $\mathrm{Cu}_{2} \mathrm{NiZn}$ investigated by Vrijen et al. [18]. However, it is a difficult task to show the existence of the $\mathrm{L}^{\prime}$ experimentally because the $\mathrm{X}$-ray reflections are the same as those of the $\mathrm{L}_{2}$ structure. Nevertheless, this structure has to be studied since it is quadratic and may lead to brittleness.

In our simplified model, all the other structures, $\mathrm{A}_{1}, \mathrm{Ll}_{2}$ and $\mathrm{Ll}_{0}$, have, of course, the same stability conditions as in pure binary alloys $\left({ }^{2}\right)$.

\section{Phase diagram.}

When the temperature is raised, the configurational entropy of the system must be taken into account. The free energy is no longer a linear form of the variables and to each set of pair energies corresponds a specific topology of the ternary phase diagram. A general classification of ternary regular solid solutions was presented by Meijering [7]. Several cases involving ordering energies were treated by several authors, however they did not include the presence of the $L^{\prime}$ phase in their investigations.

(2) The same GSA analysis applied to BCC structures interacting through a next nearest neighbour Ising model leads to the occurrence of two phases of stoechiometry $\mathrm{A}_{2} \mathrm{BC}$, namely the Heussler phase and a phase of symmetry $\mathrm{F} \overline{4} 3 \mathrm{~m}$ following that the parr $\mathrm{BC}$ orders as first or second neighbor [2]. 
In this section we study the case $\tilde{\mathbf{J}}=(1,1,1)$. This choice is apparently unrealistic, but it is expected to give insights into the topology of phase diagrams where the three binary components show ordered phases, as for example in the Ni-Pt-Fe system. The main advantage of this choice for $\mathbf{J}$ is to be symmetric with respect to exchanges of $\mathrm{A}, \mathrm{B}, \mathrm{C}$ atoms and so is the phase diagram.

As long as computation takes place in the grand canonical space, we may draw the $0 \mathrm{~K}$ phase diagram. Substituting expression (4) into equation (2), we see that with each cone vector $\nu_{t}$ Is associated a straight line in the chemical potential space of the form:

$$
\mathbf{J} \cdot \boldsymbol{v}_{1}=\mu_{1} \nu_{1}^{1}+\mu_{2} \nu_{1}^{2}+6\left(\nu_{1}^{3}+\nu_{1}^{7}+\nu_{1}^{5}\right)
$$

where $\mu_{1}$ and $\mu_{2}$ are two chemical potentials.

The results are shown in figure 2 . In this phase portrait, polygons correspond to ternary phases while semt-infinite domains are associated with edge phases (binary phases or pure elements) where the chemical potential of the vanishing element diverges. We also notice that the $\mu_{\mathrm{A}}-\mu_{\mathrm{B}}$ spacing between binary $\mathrm{A}, \mathrm{A}_{3} \mathrm{~B}$ and $\mathrm{AB}$ are the same as in the binary system. This phase diagram is very important as it gives the range of $\mu$ parameters from which free energy minimization can be undertaken.

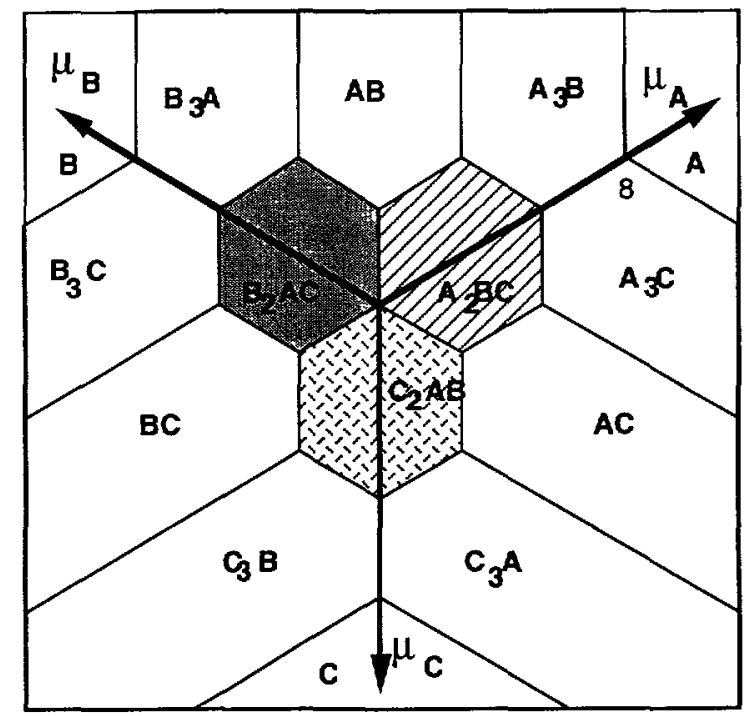

Fig. 2. - Chemical potentrals phase diagram at $T=0 \mathrm{~K}$, for the symmetrical case $\mathbf{J}=(1,1,1)$. In this phase portrait, purely ternary ground states correspond to polygons while binary ground states are associated to open domains. At the corner between $A, A_{3} B$ and $A_{3} C$ the chemical potential $\mu_{\mathrm{A}}$ equals 8 , which gives the scale of the mapping.

Before discussing the effect of composition and temperature on the boundaries of the $\mathrm{L}^{\prime}$ phase, we would like to summarize the strategy we followed in the CVM tetrahedron computations. The grand potential $\mathcal{S}$ for a given phase is written :

$$
\begin{aligned}
\mathcal{G} & =\mathfrak{U}-\mathcal{C} \&-\mathfrak{C} \cdot \boldsymbol{\mu}=\mathfrak{F}-\mathfrak{C} \cdot \boldsymbol{\mu} \\
\mathfrak{U} & =\tilde{\mathbf{J}} \cdot \mathbf{X}
\end{aligned}
$$




$$
\begin{aligned}
& \mathcal{S}=-\sum_{\alpha} a_{\alpha} \sum_{j=1}^{J=1 \alpha} \gamma_{\alpha}^{J} P_{\alpha}^{J} \ln P_{\alpha}^{J} \\
& \mathbf{P}=M \mathbf{X}
\end{aligned}
$$

where $\mathbf{X}$ is the vector whose components account for the correlation functions. The dimensionality of $\mathbf{X}$ depends on the phase under consideration, and ranges from 15 for the $\mathrm{A} 1$ phase to 81 in the less symmetric phases L" (Pmmm).

$\mathrm{C}$ accounts for the independent concentrations, linearly linked to $X(1)$ and $X(2)$.

$\alpha$ indexes the clusters and the $a_{\alpha}$ 's are the CVM coefficients. The relevant clusters are : the point, the nn pair and the tetrahedron.

The probability vector associated with a cluster $\alpha$ have components $\mathrm{P}_{\alpha}^{\prime}$ that are $\gamma_{\alpha}^{\prime}$ times degenerated.

In the chemical potential isothermal phase diagram, a three-phase equilibrium is a point and a two-phase equilibrium is a line. The corresponding features in the composition isothermal phase diagram are three-phase triangles and two-phase tie-lines respectively. If an isometric mapping is used for both concentrations and chemical potentials, we obtain an interestıng orthogonality relation between the two-phase ( 1 and 2$)$ tie-lines $\left(\mathbf{C}_{2}-\mathbf{C}_{1}\right)$ and the tangent to the chemical potential curves $(\delta \boldsymbol{\mu})$ :

$$
\left(\mathbf{C}_{2}-\mathbf{C}_{1}\right) \cdot \delta \boldsymbol{\mu}=0
$$

The computation of the ternary phase diagram consists in searching the isothermal boundaries between the available phases. The entropy is computed by minımizıng the grand canonical free energy function $\mathcal{G}$ with respect to the variables $\mathbf{X}$, the solution $\mathbf{X}^{*}(\boldsymbol{\mu}, T)$ represents the equilibrium state of order. To compute the phase boundaries, we evaluate the derivatives of $\Phi$ (taken at equilibrium) with respect to the chemical potentials, from the compositions.

$$
\left.\nabla_{\mu} \mathfrak{G}\right|_{\chi^{*}}=\left.\frac{\delta \mathfrak{G}}{\delta \boldsymbol{\mu}}\right|_{\chi^{*}}=-\left.e\right|_{\chi^{*}}
$$

Starting from an arbitrary point $\boldsymbol{\mu}$, equation (12) helps us to locate three phase equilibrium, that $1 \mathrm{~s}$, to determine the specific vectors $\boldsymbol{\mu}$ for which $\mathcal{G}$ takes the same value in the three phases. The computations are efficiently done using a Newton-like algorithm. From such a point, two phase boundaries were computed considering successive small variations of the chemical potentrals $\delta \boldsymbol{\mu}$ perpendicular to the difference of compositions between two phases ( 1 and 2) while keeping the equality of the grand potentials : $\delta\left(\mathcal{G}_{2}-\mathcal{G}_{1}\right)=-\left(\mathbf{C}_{2}-\mathbf{C}_{1}\right) \cdot \delta \boldsymbol{\mu}=0$. In this way, we have computed for several temperatures isothermal phase diagrams for the symmetric case : $J=(1,1 \quad 1)$ for which the $\mathrm{L}^{\prime}$ phases are the most stable.

For $k T / J=1$, the isothermal phase diagram is drawn in figure $3 \mathrm{a}$. This temperature is an intermediate case between $k T=0 \mathrm{~K}$ and the temperatures of the binary congruent transitions $k T_{\mathrm{c}}\left(\mathrm{Ll}_{2}\right)=194 \times J^{\mathrm{AB}}$ and $k T_{\iota}\left(\mathrm{Ll}_{0}\right)=1.89 \times J^{\mathrm{AB}}$ of the CVM tetrahedron approximation. Each ordered ground state phase remains present but a new phase which is not a ternary ground state appears at the center of the triangle. This phase has a structure we have called $\mathrm{L}^{\prime \prime}$ in which the four cubic sublattices of the FCC lattice are independent (see Tab. I). It should correspond to a ground state of a quaternary alloy $\mathrm{ABCD}$, in which the fourth element $\mathrm{D}$ is a combination of the other three species $A, B$ and $C: D=\frac{A+B+C}{3}$

Finel and Ducastelle [17] have shown that the $\mathrm{L}^{\prime}$ structure which is a ground state of a ternary alloy but not of a binary alloy may appear between the $\mathrm{Ll}_{2}$ and the $\mathrm{L} 1_{0}$ phases at low but non-zero temperatures in the binary diagram. This result is analogous to ours: the 

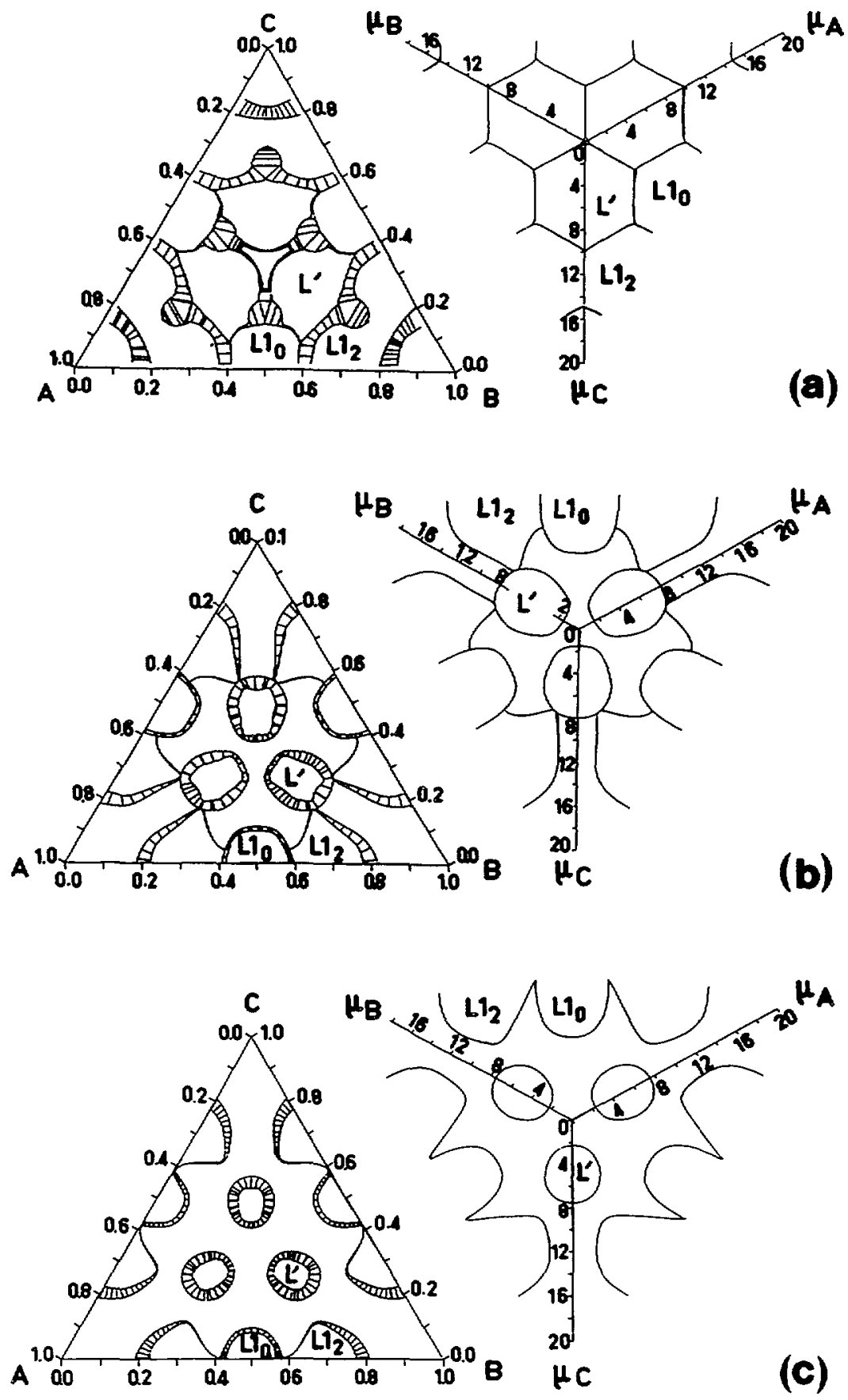

Fig. 3. - Composition and chemical potentral phase diagram for the symmetrical case : a) $k T=1$. In addition to the ground state structures, a " quaternary " phase $\mathrm{L}$ " appears in the phase diagram center. A miscibility gap exists between $\mathrm{Ll}_{2}$ phases of different composition. b) $k T=15$. The structure $L^{\prime \prime}$ have disappeared and the $L^{\prime}$ phases are remote from each other and the ordered $L^{\prime}$ and $\mathrm{L} 1_{2}$ phases separate. c) $k T=1.61$ At this temperature, there are no more ordered phases in equilibrium with each other. The $L^{\prime}$ phase is still stable in a large domain, and it will persist up to the congruent temperature $k T=174$. 
L" which is a ground state of a quaternary alloy appears in the ternary alloy phase diagram as an entropy effect.

The $\mathrm{L}^{\prime}$ phases display a broad domain of stability around the nomınal compositions $\mathrm{A}_{2} \mathrm{BC}, \mathrm{AB}_{2} \mathrm{C}$ and $\mathrm{ABC}_{2}$. The tie-lines between $\mathrm{Ll}_{2}$ and $\mathrm{L}^{\prime}$ phases are large, showing that the transition $\mathrm{Ll}_{2}-\mathrm{L}^{\prime}$ is first order whereas the tie-lines between $\mathrm{L}^{\prime}$ and $\mathrm{L} 1_{0}$ phases from large near the three phase triangle $\mathrm{L}^{\prime}-\mathrm{L}^{\prime}-\mathrm{L}_{0}$ become narrower and narrower as they approach the binary edge leading to a possible second-order transition before reaching the pure binary alloy where this transition is actually second-order according to Finel and Ducastelle [17].

There exists a continuous $\mathrm{Ll}_{2}$ phase between the binary edges $A_{3} B$ and $A_{3} C$. $A$ small miscibility gap appears in the $L 1_{2}$ phase domain between the two ordered phases. Clustering then can appear without disorder. The equilibrium of these two $\mathrm{Ll}_{2}$ phases with the $L^{\prime}$ phase leads to another rather broad three-phase domain.

It is interesting to note that the chemical phase diagram has a topology very close to that at $T=0 \mathrm{~K}$, showing once again that the chemical potentials are good variables for computations.

For $\frac{k T}{J}=1.5$ (Fig. $3 \mathrm{~b}$ ) the phase $\mathrm{L}^{\prime \prime}$ has disappeared and the $\mathrm{L}^{\prime}$ phases are remote from each other but are in equilibrium with the Al disordered phase. As can be seen in figure $3 \mathrm{~b}$, this temperature has been chosen because it corresponds to the separation of the ordered phases $L^{\prime}$ and $L l_{2}$. The binary phases $L l_{2}$ and $L l_{0}$ remain in equilibrium up to an addition of about 5 at $\%$ of the ternary element.

The temperature $\frac{k T}{J}=1.61$, corresponds to the separation of the binary phases $\mathrm{L}_{2}$ and $\mathrm{L1}_{0}$ (Fig. $3 \mathrm{c}$ ). All the ordered phases are now surrounded by the disordered phase A1. The $L^{\prime}$ phases have still a large domain of stability. The $\mathrm{L}_{2}-\mathrm{A} 1$ phase boundary is nearly tangent to the binary edge.

From figure $3 c$, we expect a dome for the $L^{\prime}$ phase, the top of which is the congruent point. Using a similar algorithm to that followed for finding the three phase domain, we have obtained the temperature of the $\mathrm{L}^{\prime}-\mathrm{A} 1$ congruent point : $k T_{\mathrm{c}}=1.74 \times J^{\mathrm{AB}}$ at the composition $A_{0492} B_{0.254} C_{0254}$. The deviation apart from the stoichiometry $A_{2} B C$ works then in the same way as for the binary congruent point $\mathrm{Ll}_{2}-\mathrm{A} 1$ occurring at composition $\mathrm{A}_{0736} \mathrm{~B}_{0264}$ : the congruent point is pulled towards the center of the diagram. The value of $T_{c}$ for $\mathrm{L}^{\prime}$ is of the same order of magnitude as the $T_{\mathrm{c}}$ of the binary phases $\mathrm{L}_{2}$ or $\mathrm{L}_{0}$, thus we expect that the $\mathrm{L}^{\prime}$ phase will not be prevented for kinetucs problems due to a too low temperature of stability. All the more, the driving force for its formation will be high enough.

\section{Conclusion.}

We have developed a new method to analyse the ground state problem. The method is based on our ability to solve homogeneous linear system of inequalities.

The method has been illustrated within the framework of the nearest neighbour Ising model Using the CVM tetrahedron approximation we have shown the existence of the ordered phase $L^{\prime}$, which can be described by using three independent sublattices on the FCC lattice. This phase, which cannot be distinguished from the $\mathrm{L}_{2}$ structure by X-ray diffraction, should be carefully studied since it is quadratic and may lead to brittleness. It is expected that $L^{\prime}$ may be identified by electron microscopy, by a careful study of the different variants.

At $0 \mathrm{~K}$, this structure is a ground state if the first three pair energies tend to order with close order of magnitude. In this case, this structure is stable for a range of compositions and temperatures very comparable to those of the $\mathrm{Ll}_{2}$ and $\mathrm{Ll}_{0}$ phases. Moreover, miscibility gaps may appear within the $\mathrm{Ll}_{2}$ ordered domains. 


\section{References}

[1] Asta M., de Fontane D., van Schilfgaarde M., Sluiter M. and Methfessel M., Phys. Rev. B 46 (1992) 5055.

[2] Rudin G.. Finel A., $J$ Phys. Condens. Matter 5 (1993) 9105.

[3] Bieber A., Gauthier F., Treglı G. and Ducastelle F., Solld State Commun. 39 (1981) 149 :

See also Bieber A. and Gauthier F., Acta Metallogr 34 (1986) 2291.

[4] Cénédèse P., Marty A. and Calvayrac Y., J. Phy's. France 50 (1989) 2193.

See also Caudron R., Sarfatı M., Barrachin M.. Finel A., Ducastelle F. and Solal F., I Phys. I France 2 (1992) 1145.

[5] Connoly J. W. and Willams A. R., Phys. Rev. B 27 (1983) 5169.

[6] Sanchez J. M., Phys. Rev. B 48 (1993) 14013.

[7] Meijering J. L., Philips Res. Rep. 6 (1951) 183.

[8] Kikuchi R., de Fontane D., Murakamı M. et Nakamura T., Acta Metallogr. 25 (1977) 207.

[9] Kikuchi R., Acta Metallogr 25 (1977) 195.

[10] Kanamori J., Prog. Th. Phys. 35 (1966) 16;

See also Kanamorı J., Solid State Commun. 50 (1984) 363;

Kanamorı J., J. Phys. Soc Jpn 52 (1984) 250.

[11] Kanamorı J. and Kaburagı M., J. Phys. Soc. Jpn 52 (1984) 4184.

[12] Allen S. M. and Cahn J. W., Acta Metallogr. 20 (1972) 423 ;

See also Allen S. M. and Cahn J. W., Scripta. Met. 7 (1973) 1261.

[13] Cahn J. W. and Kikuchi R., Acta Metallogr. 27 (1979) 1329.

[14] Finel A., Thèse de Doctorat d'Etat de I université Parıs IV, Paris (1987).

[15] Motzkin T. S., Beiträge zur Theorıe der lınearen Ungleichungen, Dissertatıon, Basel (1933);

Burger E., Über homogene lineare Ungleıchungsysteme, Z. Angew. Math. Mech. 36 (1956) 135 ;

See also, Kölbig K. S. and Schwarz F., Comp Phys. Commun. 17 (1979) 375.

[16] Minoux M., Programmation mathématique. Théone et algorithmes, Collection Technique et Scientıfıque des Télécommunications (Ed. Dunod, Parıs, 1983) p. 211.

[17] Finel A. and Ducastelle F., Europhys. Lett. 1 (1986) 135 ; Erratum, p. 543.

[18] Vrijen J., Bronsveld P., Van Der Veen J. and Radelaar S., Z. Metallkd. 67 (1976) 473. 